\title{
Long non-coding RNA LINC01006 exhibits oncogenic properties in cervical cancer by functioning as a molecular sponge for microRNA-28-5p and increasing PAK2 expression
}

\author{
LIBIN TIAN $^{1,2^{*}}$, FENG HAN $^{3 *}$, JING YANG $^{1}$, XIAOQIONG MING ${ }^{2}$ and LILI CHEN ${ }^{2}$ \\ ${ }^{1}$ Department of Obstetrics and Gynecology, Renmin Hospital of Wuhan University, Wuhan, Hubei 430060; \\ ${ }^{2}$ Department of Obstetrics and Gynecology, China Resources WISCO General Hospital, Wuhan, Hubei 430080; \\ ${ }^{3}$ Department of Respiratory Medicine, Wuhan Children's Hospital, Tongji Medical College, \\ Huazhong University of Science and Technology, Wuhan, Hubei 430015, P.R. China
}

Received December 7, 2020; Accepted January 20, 2021

DOI: $10.3892 / \mathrm{ijmm} .2021 .4879$

\begin{abstract}
As previously reported, long intergenic non-protein-coding RNA 1006 (LINC01006) plays crucial roles in prostate, pancreatic and gastric cancers. However, whether it plays important roles in cervical cancer remains unclear. The present study thus aimed to determine the precise role of LINC01006 in cervical cancer and elucidate its regulatory mechanisms. The expression of LINC01006 in cervical cancer was examined by reverse transcription-quantitative polymerase chain reaction. Cell proliferation assay, flow cytometric analysis, Transwell migration and invasion assays, and tumor xenograft model experiments were performed to elucidate the roles of LINC01006 in cervical cancer. Bioinformatics analysis, luciferase reporter assay, RNA immunoprecipitation and rescue experiments were performed for mechanistic analyses. The expression of LINC01006 was found to be upregulated in cervical cancer and to be associated with a poor prognosis. The absence of LINC01006 inhibited the proliferation, migration and invasion of cervical cancer cells, whereas it promoted cell apoptosis in vitro. The downregulation of LINC01006 impeded tumor growth in vivo. LINC01006 was verified as an endogenous 'sponge' that competed for microRNA-28-5p (miR-28-5p), which resulted in the upregulation of the miR-28-5p target P21-activated kinase 2 (PAK2). Rescue experiments revealed that the suppression of miR-28-5p expression or the overexpression of PAK2 abrogated the effects of LINC01006 downregula-
\end{abstract}

Correspondence to: Dr Jing Yang, Department of Obstetrics and Gynecology, Renmin Hospital of Wuhan University, 238 Jiefang Road, Wuhan, Hubei 430060, P.R. China

E-mail: obstetrics_yj@126.com

*Contributed equally

Key words: molecular sponge, competing endogenous RNA theory, LINC01006, cervical cancer tion on malignant cellular functions in cervical cancer. On the whole, the present study demonstrates that LINC01006 exhibits tumor-promoting functions in cervical cancer via the regulation of the miR-28-5p/PAK2 axis. These findings may provide the basis for the identification of LINC01006-targeted clinical therapy.

\section{Introduction}

Cervical cancer is one of the most frequent malignancies in gynecology, and it is the second leading cause of cancer-related mortality in women, greatly endangering their health (1). Approximately 569,847 individuals are diagnosed with cervical cancer annually, and 311,365 individuals succumb to this malignancy (2). The currently available therapies, including surgical treatment, chemotherapy and radiotherapy, effectively treat cervical cancer in situ. However, these treatment modalities have a poor therapeutic efficacy in patients with cervical cancer that is diagnosed at an advanced stage, particularly in patients with metastatic tumors (3). Notably, only approximately $40 \%$ of patients with cervical cancer survive for $>5$ years partially due to the highly invasive, uncontrolled growth and metastatic capacity of cervical cancer (4). The initiation and progression of cervical cancer involve a wide range of complex changes (5). However, the exact molecular events are largely undefined, which severely limits the exploration of novel treatment methods. Therefore, an in-depth elucidation of the mechanisms underlying the pathogenesis of cervical cancer is imperative for the development of effective therapies and improving clinical outcomes.

Long non-coding RNAs (lncRNAs) have gained increasing attention in recent years (6). These molecules are comprised of $>200$ nucleotides and are non-protein coding in nature (7). lncRNAs positively or negatively affect gene expression at the transcriptional or post-transcriptional level (8). It has been demonstrated that IncRNAs function as modulators to regulate physiological and pathological activities (9). Differentially expressed IncRNAs are observed in the majority of human diseases, including cancer (10). An increasing number of dysregulated lncRNAs have been identified in 
cervical cancer and exhibit a close association with malignant phenotypes $(11,12)$. IncRNAs play critical roles in the oncogenesis and progression of cervical cancer, and play oncogenic or antioncogenic roles in this type of cancer (13). These properties suggest that lncRNAs function as potential diagnostic biomarkers and therapeutic targets.

MicroRNAs (miRNAs or miRs) are endogenous and short RNA transcripts of approximately 22 nucleotides in length (14). These molecules play a role in post-transcriptional gene regulation by base pairing with the 3'-untranslated regions of their target genes to ultimately trigger translational suppression and/or mRNA degradation (15). The abnormal expression of miRNAs is a hallmark of cancer, including cervical cancer (16). miRNAs are crucial regulators during the genesis and development of cervical cancer $(17,18)$. The competing endogenous RNA (ceRNA) theory was introduced and it states that lncRNAs function as miRNA sponges to lower the inhibition of gene expression induced by miRNAs (19).

Previous studies have confirmed the abnormal expression of the long intergenic non-protein-coding RNA 1006 (LINC01006) in prostate (20), pancreatic (21) and gastric (22) cancers. However, whether LINC01006 plays important roles in cervical cancer remains unclear. Therefore, the present study investigated the expression status and detailed roles of LINC01006 in cervical cancer, and aimed to elucidate the mechanisms underlying the functions of LINC01006 in cervical cancer.

\section{Materials and methods}

Patient samples. The Ethics Committee of Renmin Hospital of Wuhan University approved the present study. All experiments involving human samples were performed in accordance with the principles of the Declaration of Helsinki. All participators provided written informed consent. A total of 67 pairs of cervical cancer tissues and matched adjacent normal tissues were acquired from the patients at Renmin Hospital of Wuhan University. No patient had undergone chemotherapy, radiotherapy, or other anticancer treatments prior to surgical resection. Immediately after tissue excision, all tissues were stored in liquid nitrogen until further analysis.

Cell lines. The normal human cervical epithelial cell line, Ect1/E6E7 (ATCC ${ }^{\circledR}$ CRL-2614 ${ }^{\mathrm{TM}}$ ), was purchased from the American Type Culture Collection (ATCC). Keratinocyte serum-free medium (Gibco; Thermo Fisher Scientific, Inc.) containing $0.1 \mathrm{ng} / \mathrm{ml}$ human recombinant epidermal growth factor, $0.05 \mathrm{mg} / \mathrm{ml}$ bovine pituitary extract and $0.4 \mathrm{mM}$ calcium chloride was used to culture the Ect1/E6E7 cells. In addition, 4 cervical cancer cell lines, SiHa (TCHu113), CaSki (TCHu137), C33A (TCHu176) and HeLa (TCHu187), were acquired from the Cell Bank of the Chinese Academy of Sciences. The SiHa, CaSki and HeLa cells were grown in RPMI-1640 medium (Gibco; Thermo Fisher Scientific, Inc.) supplemented with $10 \%$ fetal bovine serum (FBS; Gibco; Thermo Fisher Scientific, Inc.) and $1 \%$ penicillin-streptomycin (Gibco; Thermo Fisher Scientific, Inc.). The C33A cells were cultured in minimum essential medium (Gibco; Thermo Fisher Scientific, Inc.) with $10 \%$ FBS. All cells were routinely grown at $37^{\circ} \mathrm{C}$ in a humidified atmosphere supplied with $5 \% \mathrm{CO}_{2}$.
Cell transfection. To silence LINC01006, specific small interfering RNAs (siRNAs) targeting LINC01006 (si-LINC01006) were devised and synthesized by Shanghai GenePharma Co., Ltd. The si-LINC01006\#1 sequence was 5'-CGCAAAGTT TTCCTATTAACTCT-3'; the si-LINC01006\#2 sequence was 5'-TTCAAATTTTGACTTATTTTACA-3'; and the si-NC sequence was 5'-CACGATAAGACAATGTATTT-3'. A nonsense sequence (si-NC) was used as the negative control (NC). The miR-28-5p mimic, NC mimic, miR-28-5p inhibitor (anti-miR-28-5p) and NC inhibitor (anti-NC) were obtained from RiboBio Co., Ltd. To overexpress P21-activated kinase 2 (PAK2), the sequences of PAK2 were inserted into the pcDNA3.1 plasmid to obtain the PAK2 overexpression plasmid pcDNA3.1-PAK2 (pc-PAK2; Shanghai GenePharma Co., Ltd.). Cervical cancer cells were cultivated in 6-well plates. As per the manufacturer's instructions, cells were transfected with siRNAs (100 pmol), mimics (100 pmol), miRNA inhibitors (100 pmol) or plasmids (4 $\mu \mathrm{g})$ using Lipofectamine 2000 (Invitrogen; Thermo Fisher Scientific, Inc.).

Reverse transcription-quantitative polymerase chain reaction $(R T-q P C R)$. TRIzol reagent (Beyotime Institute of Biotechnology) was used to extract total RNA, followed by the assessment of RNA concentration and purity using a NanoDrop 2000 spectrometer (Thermo Fisher Scientific, Inc.). To determine miRNA expression, reverse transcription was performed using the Mir-X miRNA First-Strand Synthesis kit (cat. no. 638315; Takara Biotechnology Co., Ltd.). Using complementary DNA (cDNA) as a template, the Mir-X miRNA qRT-PCR TB Green ${ }^{\circledR}$ kit (cat. no. 638314; Takara Biotechnology Co., Ltd.) was used to perform qPCR. U6 small nuclear RNA was used as the internal control. To quantify the mRNA expression levels of LINC01006 and PAK2, cDNA was obtained by performing reverse transcription using the PrimeScript ${ }^{\mathrm{TM}}$ RT Reagent klit with gDNA Eraser (cat. no. RR047A; Takara Biotechnology Co.). Thereafter, PCR amplification was performed using TB Green Premix Ex Taq (cat. no. RR420A; Takara Biotechnology Co.). The mRNA expression levels of LINC01006 and PAK2 were normalized to glyceraldehyde 3-phosphate dehydrogenase (GAPDH). Gene expression was calculated using the $2^{-\Delta \Delta \mathrm{Cq}}$ method (23). The sequences of all primers are presented in Table I.

Cell proliferation assay. Transfected cells were collected after $24 \mathrm{~h}$, and the cell number was counted using a blood cell counting chamber. Cells were resuspended in $10 \%$ FBS-supplemented culture medium. A $100-\mu 1$ cell suspension containing $2 \times 10^{3}$ cells was seeded into 96 -well plates, followed by culturing in the aforementioned conditions for different time periods of $0,24,48$ and $72 \mathrm{~h}$. For the cell proliferation assay, $10 \mu \mathrm{l}$ of cell counting kit-8 (CCK-8) reagent were added to each well, followed by incubation for a further $2 \mathrm{~h}$ at $37^{\circ} \mathrm{C}$. The absorbance was measured at a wavelength of $450 \mathrm{~nm}$ using a Multiskan Spectrum Microplate spectrophotometer (Thermo Fisher Scientific, Inc.).

Flow cytometric analysis. After $48 \mathrm{~h}$, the transfected cells were digested with $0.25 \%$ trypsin and collected following centrifugation at $1,000 \mathrm{xg}$ for $5 \mathrm{~min}$ at room temperature. As per the instructions of the Annexin V-FITC Apoptosis Detection kit 
Table I. Primer sequences used for RT-qPCR.

\begin{tabular}{ll} 
Gene & \\
\hline LINC01006 & \multicolumn{1}{c}{ Sequences $\left(5^{\prime} \rightarrow 3^{\prime}\right)$} \\
PAK2 & $\begin{array}{l}\text { Forward: GTGTGACACATCGGAGTGAATTGAG } \\
\text { Reverse: AACCCTGCACTATTTGTGGCGT }\end{array}$ \\
GAPDH & Forward: ATAACGGAGAACTGGAAGATAAGC \\
& Reverse: AGATGATTTTATGCCTGGGCTT \\
U6 & Forward: ACCTGACCTGCCGTCTAGAAAA \\
hsa-miR-28-5p & Reverse: TTGAAGTCAGAGGAGACCACCTG \\
hsa-miR-154-3p & Forward: CTCGCTTCGGCAGCACA \\
& Reverse: AACGCTTCACGAATTTGCGT \\
& Forward: TCGGCAGGAAGGAGCUCACAGUC \\
& Reverse: CACTCAACTGGTGTCGTGGA \\
& Forward: TCGGCAGGGCUUCCGUUGUGCC
\end{tabular}

(Beyotime Institute of Biotechnology), the harvested cells were resuspended in $195 \mu \mathrm{l}$ Annexin V-FITC buffer, followed by probing with $5 \mu \mathrm{l}$ of Annexin V-FITC and $10 \mu \mathrm{l}$ of propidium iodide. Following $20 \mathrm{~min}$ of incubation at $20-25^{\circ} \mathrm{C}$ in the dark, apoptosis was detected using a FACSCalibur flow cytometer (BD Biosciences).

Transwell migration and invasion assays. For the migration assay, transfected cells were treated with $0.25 \%$ trypsin, rinsed with phosphate-buffered saline, and centrifuged at $1,000 \mathrm{x} \mathrm{g}$ for $5 \mathrm{~min}$ at room temperature. The collected cells were resuspended in culture medium without FBS. The upper chambers (8 $\mu \mathrm{m}$ pores; Corning Inc.) were covered with $100 \mu \mathrm{l}$ of the cell suspension containing $5 \times 10^{4}$ cells. The lower chamber included $20 \%$ FBS-supplemented culture medium, followed by incubation at $37^{\circ} \mathrm{C}$ for $24 \mathrm{~h}$. The cells in the inner membrane were cleaned, and the cells that passed through the pores were fixed with $100 \%$ methanol and dyed with $0.1 \%$ crystal violet (Beyotime Institute of Biotechnology) at room temperature for $30 \mathrm{~min}$. The number of migrated cells was counted under a light microscope (Olympus Corporation). A total of 5 visuals were randomly selected for microscopic observation. Cell invasion was examined using the same experimental procedures, with the exception that the chambers were precoated with Matrigel (BD Biosciences).

Tumor xenograft model. To inhibit LINC01006 expression, short hairpin RNAs (shRNAs) against LINC01006 (sh-LINC01006) and NC shRNA (sh-NC) were constructed and synthesized by Shanghai GenePharma Co., Ltd. The sh-LINC01006 sequence was 5'-CCGGTTCAAATTTTGACTTATTTTACACTCGAG TGTAAAATAAGTCAAAATTTGAATTTTTG-3'; and the sh-NC sequence was 5'-CCGGCACGATAAGACAATGTATTT CTCGAGAAATACATTGTCTTATCGTGTTTTTG-3'. The synthesized shRNAs were inserted into the pLKO.1 vector (Biosettia, Inc.) and transfected into 293T cells (Cell Bank of Chinese Academy of Sciences) in parallel with psPAX2 and pMD2.G. Following 2 days of incubation at $37^{\circ} \mathrm{C}$, HeLa cells were transfected with lentiviruses expressing sh-LINC01006 or sh-NC. The infected HeLa cells were incubated with puromycin
(5 $\mu \mathrm{g} / \mathrm{ml}$; Sigma-Aldrich; Merck KGaA) to select stable cell lines.

The Institutional Animal Care and Use Committee of Renmin Hospital of Wuhan University approved the experimental steps involving animals. A total of $6 \mathrm{BALB} / \mathrm{c}$ female nude mice (4-5 weeks old; weighing 20 g; Shanghai Laboratory Animal Center of Chinese Academy of Sciences) were subcutaneously injected with HeLa cells that were stably transfected with sh-LINC01006 or sh-NC. Each group contained 3 nude mice. The animals were kept under specific pathogen-free conditions at $25^{\circ} \mathrm{C}$ and $50 \%$ humidity, with a 10:14 light/dark cycle and ad libitum access to food and water. The volume of the tumor xenografts was monitored weekly and recorded. Tumor volume was determined using the following formula: Volume $=0.5 \mathrm{x}\left(\right.$ length $\mathrm{x}$ width $\left.{ }^{2}\right)$. The mice were euthanized by means of cervical dislocation at week 4 , and tumor xenografts were obtained, imaged and weighed.

Subcellular fractionation assay. The Cytoplasmic and Nuclear RNA Purification kit (Norgen Biotek Corp.) was used to prepare the cytoplasmic and nuclear fractions of the cervical cancer cells. The RNA of both fractions was used to determine the LINC01006 distribution by performing RT-qPCR. GAPDH and U6 were considered the cytoplasmic and nuclear internal references, respectively.

Bioinformatics analysis and luciferase reporter assay. The binding sequences between miR-28-5p and LINC01006 were predicted using StarBase 3.0 (http://starbase.sysu.edu.cn/). TargetScan (http://www.targetscan.org/vert_60/) and miRDB (http://mirdb.org/miRDB/index.html) were used to search for the putative targets of miR-28-5p. The Cancer Genome Atlas (TCGA) database was applied to analyze LINC01006 expression in cervical squamous cell carcinoma and endocervical adenocarcinoma (CESC).

LINC01006 fragments containing the wild-type (WT) or mutant (MUT) miR-28-5p binding sites were amplified by Shanghai GenePharma Co., Ltd. and fused into the psiCHECK ${ }^{\mathrm{TM}}-2$ luciferase reporter vector (Promega Corporation). The obtained luciferase reporter vectors 
were labeled WT-LINC01006 and MUT-LINC01006. The WT-PAK2 and MUT-PAK2 reporter vectors were produced in a similar manner. For the reporter assay, the synthesized reporter vectors $(0.2 \mu \mathrm{g})$ were co-transfected with miR-28-5p mimic (20 pmol) or NC mimic (20 pmol) into cervical cancer cells. Luciferase activity was detected at $48 \mathrm{~h}$ following transfection using the Dual-Luciferase Reporter Analysis system (Promega Corporation). Renilla luciferase activity was used for the Firefly luciferase activity normalization.

RNA immunoprecipitation (RIP) assay. RIP assay was performed using the Magna RIP RNA-Binding Protein Immunoprecipitation kit (cat. no. 03-110; Merck-Millipore). The lysate of cervical cancer cells was obtained by cultivating the cells with RIP lysis buffer, followed by the addition of magnetic beads conjugated with human anti-argonaute 2 (Ago2) or control anti-IgG antibodies (1:5,000 dilution; both from cat. no. 03-110; Merck Millipore). Following overnight incubation at $4^{\circ} \mathrm{C}$, the magnetic beads were rinsed with washing buffer. After probing with proteinase $\mathrm{K}$, the relative enrichment of LINC01006, miR-28-5p, and PAK2 in the immunoprecipitate was assessed by RT-qPCR.

Western blot analysis. RIPA lysis buffer (Beyotime Institute of Biotechnology) was used to extract total proteins from the cells. Total protein concentration was quantified using the Pierce $^{\mathrm{TM}}$ bicinchoninic acid Protein Assay kit (cat. no. 23225; Thermo Fisher Scientific, Inc.). The equivalent protein (30 $\mu \mathrm{g})$ was separated using $10 \%$ sodium dodecyl sulfate-polyacrylamide gel electrophoresis. The resolved proteins were electrotransferred onto polyvinylidene fluoride membranes. Membrane blocking was performed using 5\% defatted milk powder at room temperature for $2 \mathrm{~h}$, followed by incubation with primary antibodies overnight at $4^{\circ} \mathrm{C}$. Primary antibodies specific for PAK2 (cat. no. ab76293; 1:1,000 dilution) or GAPDH (cat. no. ab181603; 1:1,000 dilution) were purchased from Abcam. After the membranes were incubated with the horseradish peroxidase-conjugated anti-rabbit secondary antibody (cat. no. ab205718; 1:5,000 dilution; Abcam) at room temperature for $2 \mathrm{~h}$, imprinting was performed using Pierce $^{\mathrm{TM}}$ ECL Western blotting Substrate (Pierce; Thermo Fisher Scientific, Inc.). Densitometry of the protein signals was implemented utilizing Quantity One software version 4.62 (Bio-Rad Laboratories, Inc.).

Statistical analysis. Experimental data are presented as the means \pm standard deviation. A Student's t-test was used to determine differences between 2 groups, and multiple group comparisons were performed using one-way analysis of variance with Tukey's post hoc test. Gene expression correlations were examined using Pearson's correlation coefficient. Kaplan-Meier survival curves and the log rank test were used for survival analyses. $\mathrm{P}<0.05$ indicated a statistically significant difference.

\section{Results}

LINC01006 interference induces cell apoptosis and restrains the proliferation, migration, and invasion of cervical cancer cells. To determine the expression pattern of LINC01006 in cervical cancer, its expression levels in CESC were examined using The Cancer Genome Atlas (TCGA) database. The expression level of LINC01006 was higher in CESC tissues than in normal tissues (Fig. 1A). Consistently, the expression level of LINC01006 was higher in cervical cancer tissues than in adjacent normal tissues (Fig. 1B). RT-qPCR was then performed to determine the expression level of LINC01006 in cervical cancer cell lines. Compared to the Ect1/E6E7 cells, a relatively higher LINC01006 expression level was confirmed in the 4 tested cervical cancer cell lines (Fig. 1C). The association between LINC01006 expression and the overall survival of patients with cervical cancer was also addressed. Patients with cervical cancer who exhibited a high LINC01006 expression had a shorter overall survival than patients who exhibited a low LINC01006 expression (Fig. 1D).

Of the 4 cervical cancer cell lines, the SiHa and HeLa cells exhibited a relatively higher LINC01006 expression. Therefore, these cells were selected to determine the specific functions of LINC01006. The SiHa and HeLa cells were transfected with si-LINC01006. To avoid off-target effects, 2 siRNAs were used, and the efficiency of RNA interference was assessed by RT-qPCR (Fig. 1E). Cell proliferation assay then demonstrated that the proliferation of the SiHa and HeLa cells was suppressed following the knockdown of LINC01006 (Fig. 1F). Flow cytometric analysis further demonstrated that the knockdown of LINC01006 promoted the apoptosis of the SiHa and HeLa cells (Fig. 1G). In addition, si-LINC01006 evidently decreased the migration (Fig. 1H) and invasion (Fig. 1I) of the $\mathrm{SiHa}$ and HeLa cells. Taken together, these results suggest that LINC01006 is upregulated in cervical cancer and functions as a promoter of cancer progression.

LINC01006 directly interacts with miR-28-5p and functions as a miR-28-5p sponge. To determine the downstream mechanism of LINC01006, the cellular localization of LINC01006 in cervical cancer cells was analyzed using a subcellular fractionation assay. The data confirmed that LINC01006 was a cytoplasmic lncRNA in cervical cancer (Fig. 2A), which suggests that it functions as a molecular sponge for miRNA and plays its pro-oncogenic roles at the post-transcription level. A search of StarBase 3.0 revealed a total of 30 miRNAs (Fig. 2B) that included binding sites for LINC01006. The analysis of the TCGA database identified 2 miRNAs (miR-28-5p and miR-154-3p) that were weakly expressed (Fig. 2C), and 7 miRNAs that were overexpressed in CESC tissues (data not shown). miR-28-5p and miR-154-3p were selected for further experiments. The expression of miR-28-5p and miR-154-3p was detected in cervical cancer cells in which LINC01006 was silenced. miR-28-5p expression increased prominently in the $\mathrm{SiHa}$ and HeLa cells following the silencing of LINC01006; however, there was no difference in the levels of miR-154-3p (Fig. 2D). The expression of miR-28-5p was downregulated in cervical cancer tissues (Fig. 2E) and Pearson's correlation coefficient revealed that the expression of miR-28-5p in cervical cancer tissues inversely correlated with that of LINC01006 (Fig. 2F). Notably, miR-28-5p and LINC01006 were enriched in Ago2-containing immunoprecipitate complexes compared to IgG control immunoprecipitate complexes (Fig. 2G). The direct binding between LINC01006 and miR-28-5p (Fig. 2H) was confirmed by luciferase reporter assay. The outcomes revealed that miR-28-5p overexpression led to a notable 
A

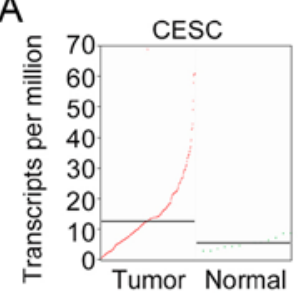

D

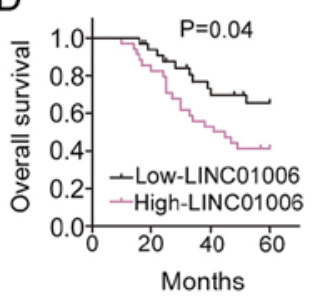

B

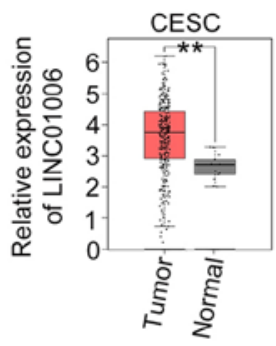

E

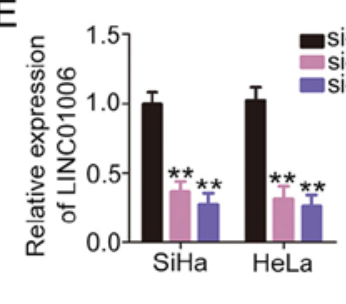

C

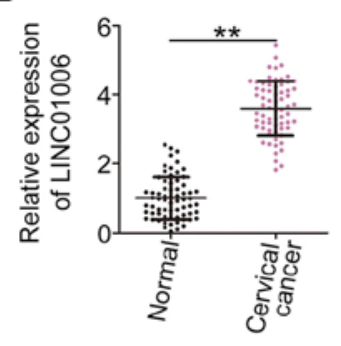

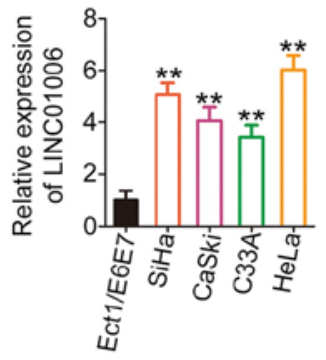

G

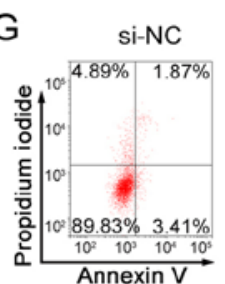

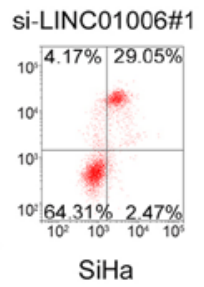

$\mathrm{SiHa}$

$\mathrm{H}$
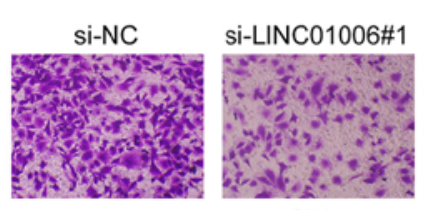

$\mathrm{SiHa}$

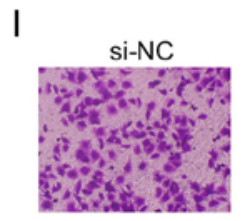

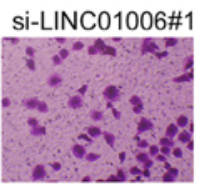

$\mathrm{SiHa}$

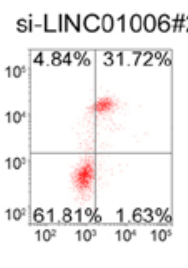

$10^{3}$
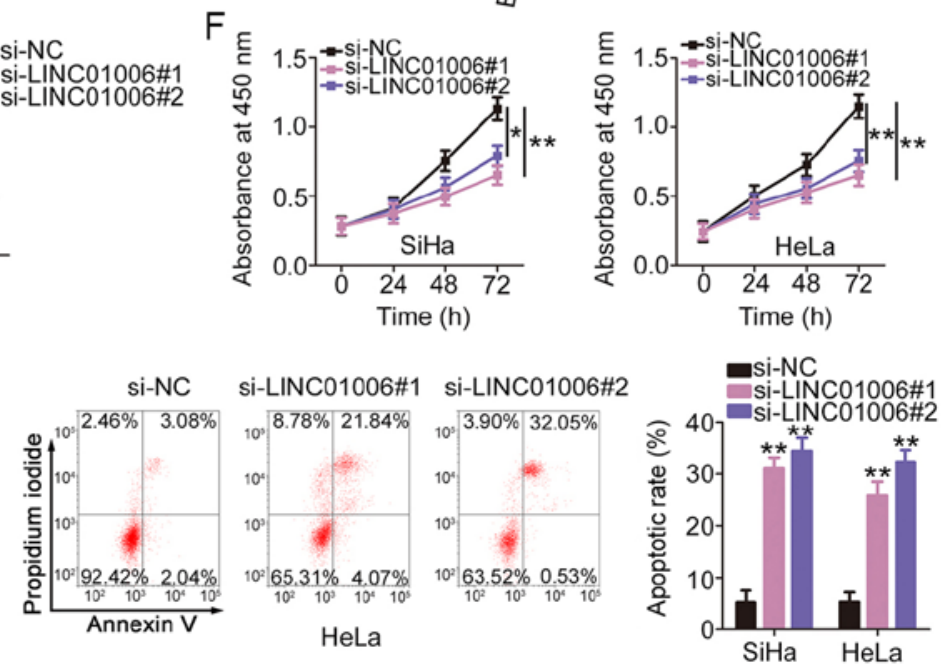

HeLa
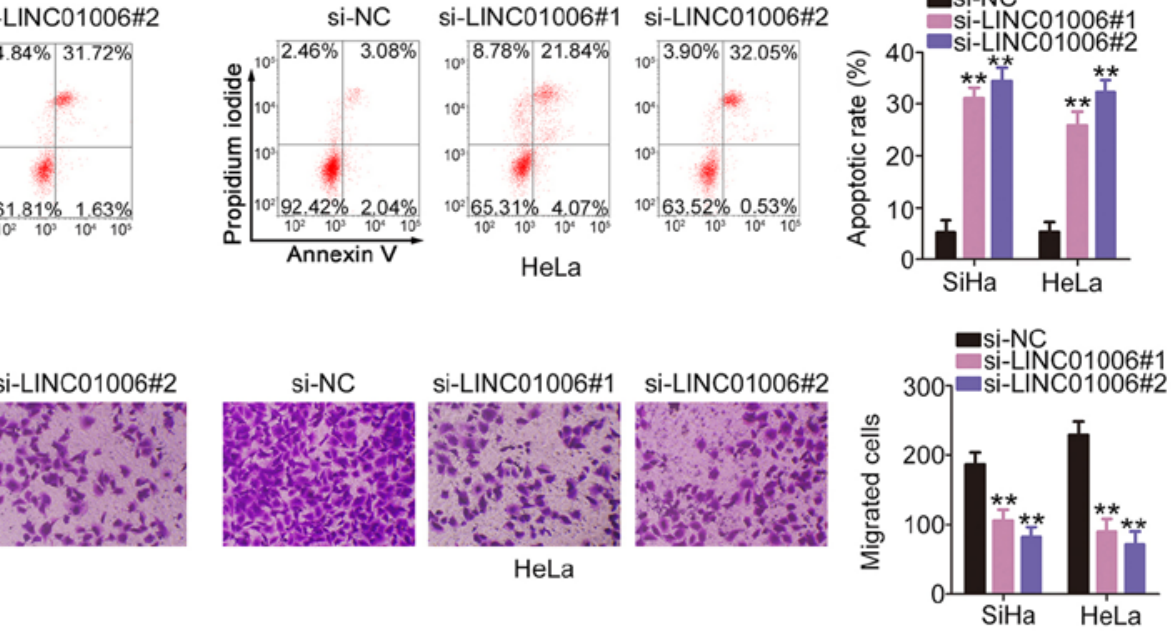

HeLa
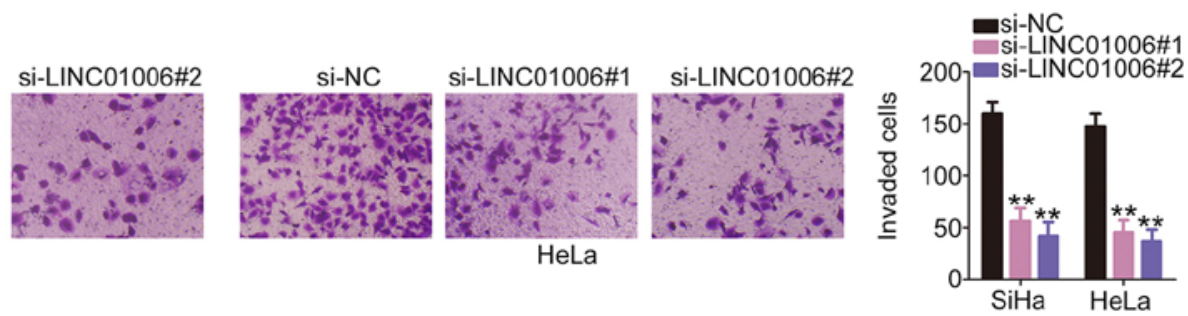

Figure 1.LINC01006 knockdown inhibits the proliferation, migration and invasion of cervical cancer cells and promotes cell apoptosis in vitro. (A) LINC01006 expression in cervical squamous cell carcinoma and endocervical adenocarcinoma was examined using The Cancer Genome Atlas (TCGA) database. (B) RT-qPCR was performed to monitor LINC01006 expression in 67 pairs of cervical cancer tissues and matched adjacent normal tissues. (C) LINC01006 expression in cervical cancer cell lines was analyzed by RT-qPCR. The normal human cervical epithelial cell line, Ect1/E6E7, was used as the control. (D) Kaplan-Meier analysis illustrated the association between LINC01006 expression and the overall survival of patients with cervical cancer. (E) The silencing efficiency of si-LINC01006 was analyzed in cervical cancer cells by RT-qPCR. (F) The proliferation of SiHa and HeLa cells was assessed using the cell proliferation assay following the silencing LINC01006. (G) Flow cytometric analysis was performed to detect the apoptotic rate of SiHa and HeLa cells after LINC01006 knockdown. (H and I) Transwell migration and invasion assays were performed to determine the migration and invasion of SiHa and HeLa cells following LINC01006 knockdown. ${ }^{*} \mathrm{P}<0.05$ and $^{* *} \mathrm{P}<0.01$ vs. respective control. LINC01006, long intergenic non-protein-coding RNA 1006.

decrease in the luciferase activity of WT-LINC01006 (Fig. 2I). However, no evident change was identified in the cells transfected with MUT-LINC01006. Taken together, these results suggest that LINC01006 directly interacts with miR-28-5p and functions as an miR-28-5p sponge in cervical cancer.

miR-28-5p is a tumor-suppressor in cervical cancer. To determine the contribution of miR-28-5p, functional changes in cervical cancer cells were examined following the overex- pression of miR-28-5p (Fig. 3A). The increased expression of miR-28-5p clearly hindered the proliferation (Fig. 3B) and promoted the apoptosis (Fig. 3C) of the $\mathrm{SiHa}$ and HeLa cells, as demonstrated in by cell proliferation assay and flow cytometric analysis. In addition, the migratory (Fig. 3D) and invasive (Fig. 3E) abilities of the $\mathrm{SiHa}$ and HeLa cells were inhibited with the enforced expression of miR-28-5p. Taken together, these results suggest that miR-28-5p exerts antioncogenic effects in cervical cancer cells. 


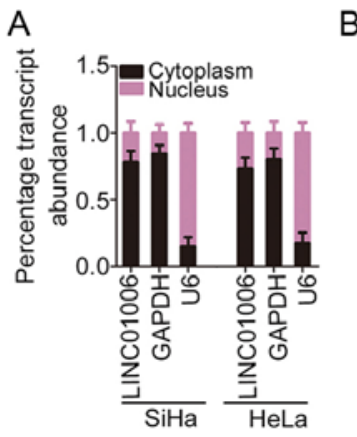

B Potential miRNAs targeting LINC01006
\begin{tabular}{|l|l|l|}
\hline miR-1185-5p & miR-2682-5p & miR-4739 \\
miR-129-1-3p & miR-28-5p & miR-4756-5p \\
miR-129-2-3p & miR-3139 & miR-487a-3p \\
miR-1321 & miR-34a-5p & miR-503-5p \\
miR-1343-3p & miR-34b-5p & miR-516a-5p \\
miR-148a-3p & miR-34c-5p & miR-556-5p \\
miR-148b-3p & miR-3679-5p & miR-624-5p \\
miR-152-3p & miR-449a & miR-671-5p \\
miR-154-3p & miR-449b-5p & miR-6783-3p \\
miR-2467-3p & miR-449c-5p & miR-708-5p \\
\hline
\end{tabular}

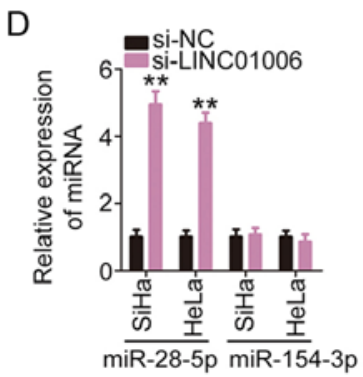

$\mathrm{H}$

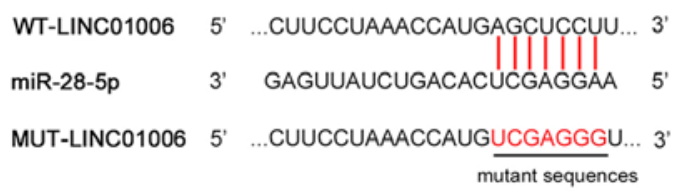

C
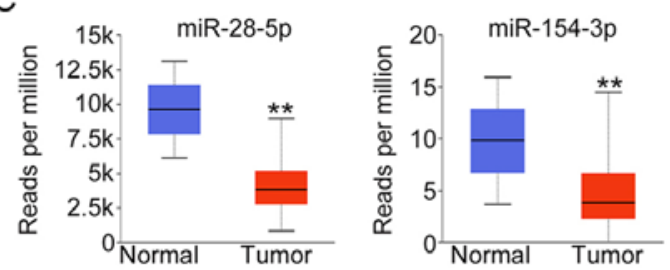

G
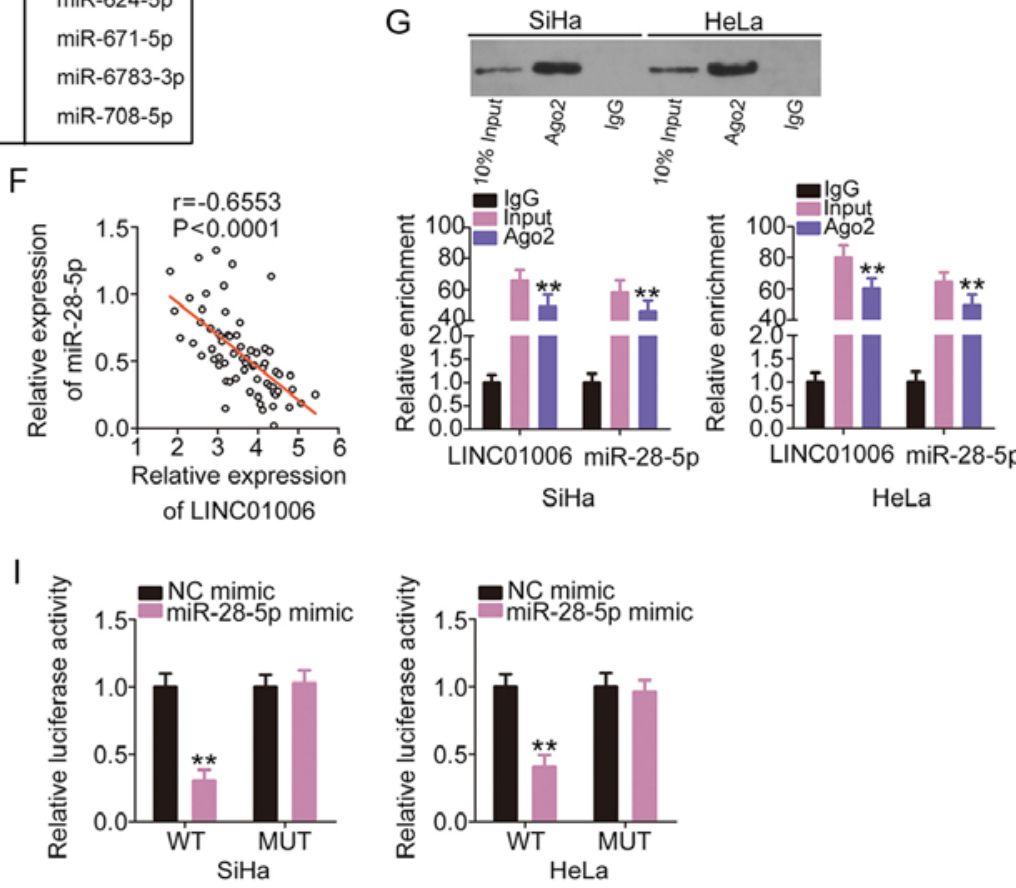

Figure 2. LINC01006 serves as a miR-28-5p sponge in cervical cancer. (A) A subcellular fractionation assay was performed to assess the location of LINC01006 in cervical cancer cells. (B) A total of 30 miRNAs had binding sites for LINC01006. (C) The expression levels of miR-28-5p and miR-154-3p in cervical cancer tissues were examined using The Cancer Genome Atlas (TCGA) database. (D) The expression of miR-28-5p and miR-154-3p in LINC01006-depleted SiHa and HeLa cells was determined by RT-qPCR. (E) RT-qPCR was performed to confirm the expression of miR-28-5p in cervical cancer tissues. (F) The correlation between LINC01006 and miR-28-5p in cervical cancer tissues was determined using Pearson's correlation coefficient. (G) RIP assay was performed to analyze the interaction between LINC01006 and miR-28-5p; the relative enrichment of LINC01006 and miR-28-5p in the immunoprecipitate was detected by RT-qPCR. (H) Putative binding sequences between LINC01006 and miR-28-5p were predicted using StarBase 3.0. The mutated site is also shown. (I) SiHa and HeLa cells were transfected with WT-LINC01006 or MUT-LINC01006 in combination with miR-28-5p mimic or NC mimic. Luciferase activity was quantified at $48 \mathrm{~h}$ following transfection. ${ }^{* *} \mathrm{P}<0.01 \mathrm{vs}$. respective control. LINC01006, long intergenic non-protein-coding RNA 1006. RIP, radioimmunoprecipitation.

LINC01006 positively regulates PAK2 in cervical cancer by acting as a decoy to miR-28-5p. Online bioinformatics prediction databases were used to determine the potential target of miR-28-5p and PAK2 (Fig. 4A) was selected for subsequent confirmation due to its critical roles in carcinogenesis and cancer progression. A luciferase reporter assay was performed to demonstrate the direct binding of miR-28-5p and PAK2. miR-28-5p did not bind MUT-PAK2 or influence its luciferase activity. By contrast, the luciferase activity of WT-PAK2 was evidently decreased in the $\mathrm{SiHa}$ and HeLa cells co-transfected the miR-28-5p mimic (Fig. 4B). miR-28-5p overexpression evidently diminished the mRNA (Fig. 4C) and protein (Fig. 4D) expression levels of PAK2 in the SiHa and HeLa cells.

Subsequent analyses were performed to determine the association between LINC01006 and miR-28-5p in PAK2 regulation. The regulatory effects of LINC01006 on PAK2 expression in cervical cancer cells were determined by RT-qPCR and western blot analysis. Notably, the knockdown of LINC01006 clearly decreased PAK2 expression in the $\mathrm{SiHa}$ and HeLa cells at the mRNA (Fig. 4E) and protein (Fig. 4F) levels, and co-transfection with anti-miR-28-5p counteracted these inhibitory effects (Fig. 4G and H). LINC01006, miR-28-5p and PAK2 were abundant in the RNA immunoprecipitated with anti-Ago2 antibody (Fig. 4I), which suggests that these three molecules co-exist in the same RNA-induced silencing complex. Compared to the adjacent normal tissues, the cervical cancer tissues had a higher PAK2 expression (Fig. 4J). PAK2 expression negatively correlated with miR-28-5p expression (Fig. 4K) and positively correlated with LINC01006 expression (Fig. 4L) in the cervical cancer tissues. Taken together, these results suggest that LINC01006 functions as a ceRNA for miR-28-5p and positively regulates PAK2 expression in cervical cancer.

LINC01006 drives the oncogenicity of cervical cancer via the miR-28-5p/PAK2 axis. Rescue experiments were performed to determine whether LINC01006 achieved its tumor-promoting roles in cervical cancer cells by affecting the miR-28-5p/PAK2 axis. RT-qPCR was performed to examined the transfection efficiency of anti-miR-28-5p. Transfection with anti-miR-28-5p resulted in a marked decrease in miR-28-5p expression in the $\mathrm{SiHa}$ and HeLa cells (Fig. 5A). Anti-miR-28-5p or anti-NC and 

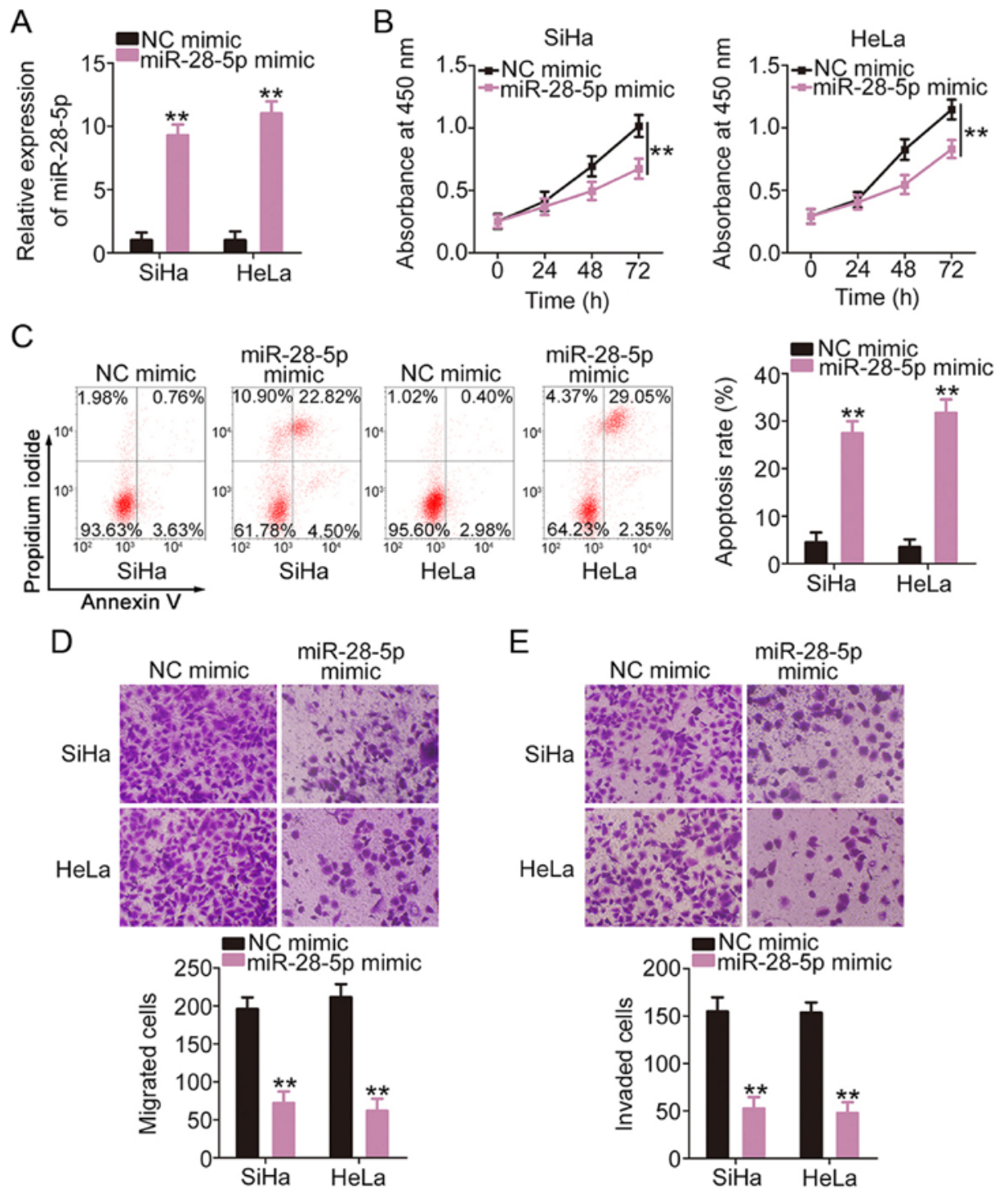

Figure 3. miR-28-5p functions as an antioncogenic miRNA in cervical cancer. (A) SiHa and HeLa cells were transfected with an miR-28-5p mimic or NC mimic. The transfection efficiency was assessed by RT-qPCR. (B and C) Cell proliferation assay and flow cytometric analysis revealed the proliferation and apoptosis of $\mathrm{SiHa}$ and HeLa cells, respectively, in the presence of miR-28-5p overexpression. (D and E) Transwell migration and invasion assays revealed the migration and invasion of $\mathrm{SiHa}$ and HeLa cells with upregulated miR-28-5p. ${ }^{* *} \mathrm{P}<0.01$ vs. respective control.

si-LINC01006 were transfected into the SiHa and HeLa cells. The loss of LINC01006 evidently restricted the proliferation (Fig. 5B) and promoted the apoptosis (Fig. 5C) of the SiHa and HeLa cells. Anti-miR-28-5p co-transfection counteracted the regulatory effects. LINC01006 interference induced a significant decrease in cell migration (Fig. 5D) and invasion (Fig. 5E), which was abolished by miR-28-5p inhibition.

The protein levels of PAK2 in the pcDNA3.1-transfected and pc-PAK2-transfected SiHa and HeLa cells were determined by western blot analysis. The data confirmed that PAK 2 protein was markedly overexpressed in the SiHa and HeLa cells following pc-PAK2 transfection (Fig. 6A). PAK2 upregulation recovered cell proliferation which was impaired due to the silencing of LINC01006 (Fig. 6B). PAK2 overexpression attenuated the promoting effect of si-LINC01006 on cell apoptosis (Fig. 6C). The re-introduction of PAK2 also restored the cell migration (Fig. 6D) and invasive (Fig. 6E) abilities that were impaired by si-LINC01006. Therefore, the miR-28-5p/PAK2 axis may act as a downstream effector of LINC01006 in cervical cancer.
Depletion of LINC01006 inhibits tumor growth in vivo. HeLa cells that were stably transfected with sh-LINC01006 or sh-NC were subcutaneously injected into nude mice to establish a tumor xenograft model. Tumor growth was evidently lower in mice in the sh-LINC01006 group than the sh-NC group (Fig. 7A). The volume (Fig. 7B) and weight (Fig. 7C) of the tumor xenografts were evidently decreased in the LINC01006-silenced group compared to the sh-NC group. The levels of LINC01006, miR-28-5p and PAK2 were analyzed in the tumor tissues. The expression of LINC01006 (Fig. 7D) and PAK2 (Fig. 7E) was downregulated, and miR-28-5p (Fig. 7F) was overexpressed in the tumor xenografts of the LINC01006 deficiency group. Overall, these results suggest that LINC01006 depletion decreases tumor growth in vivo.

\section{Discussion}

Dysfunctional lncRNAs are frequently observed in cervical cancer. These molecules have a close association with cervical 
A

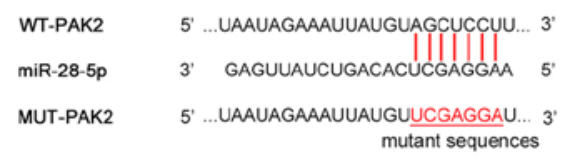

C

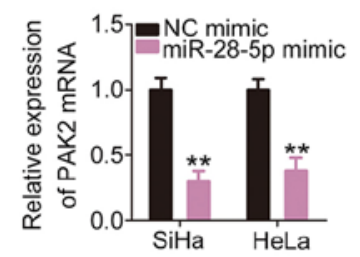

$\mathrm{F}$

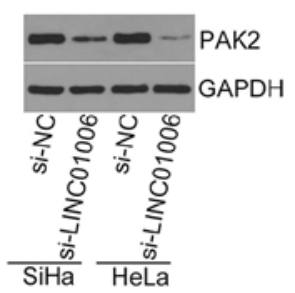

$B \geq$

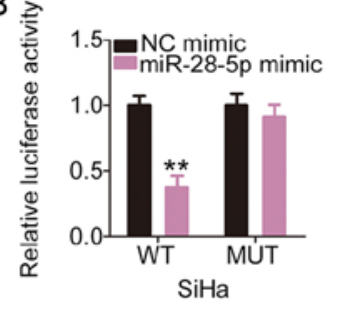

D

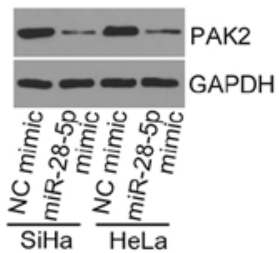

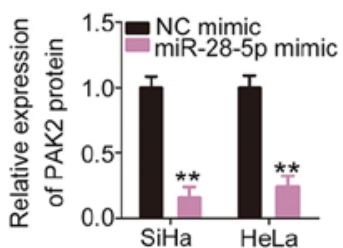

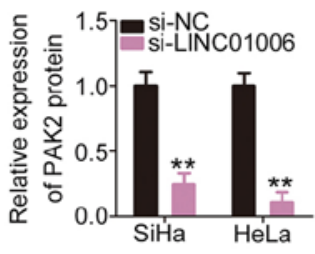

G

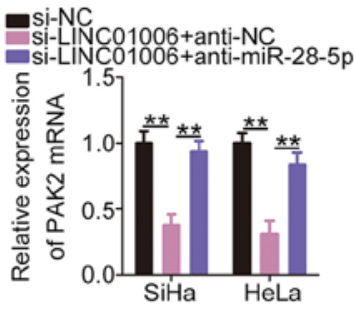

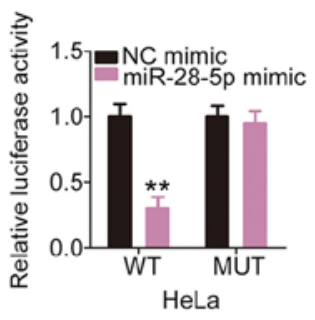

$\mathrm{E}$

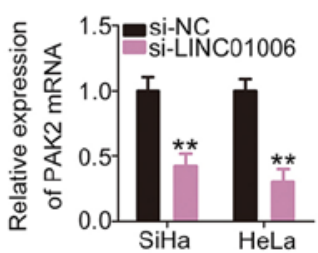

$\mathrm{H}$

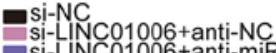

Si-LINC01006+anti-miR-28-5p

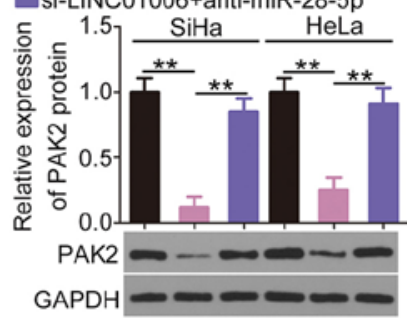

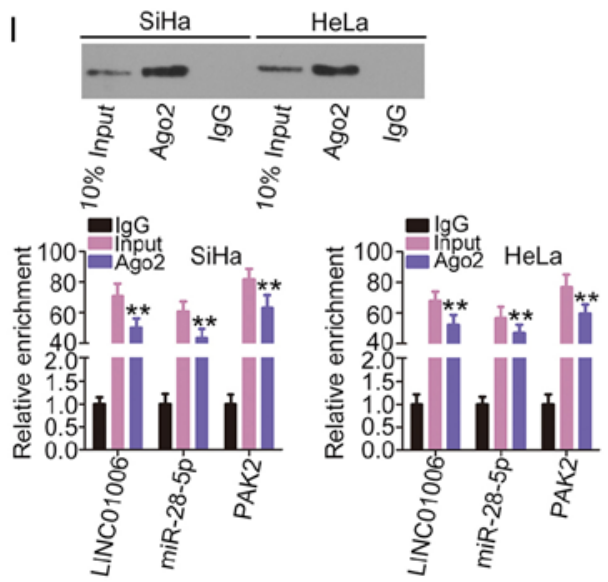
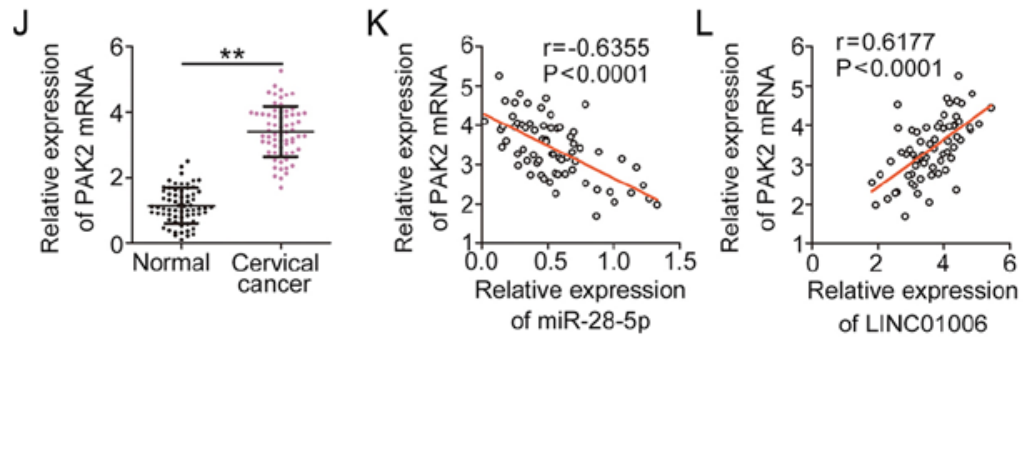

Figure 4. miRNA-28-5p directly targets P21-activated kinase 2 (PAK2) in cervical cancer. (A) Bioinformatics analysis showing the binding site between miR-28-5p and the 3'-untranslated region of PAK2. The mutated binding sequences are also shown. (B) Luciferase activity was detected in SiHa and HeLa cells after transfection with WT-PAK2 or MUT-APK2 together with miR-28-5p mimic or NC mimic. (C and D) RT-qPCR and western blot analysis were performed to detect the mRNA and protein expression levels of PAK2 in SiHa and HeLa cells when miR-28-5p was overexpressed. (E and F) The mRNA and protein expression levels of PAK2 were determined by RT-qPCR and western blot analysis, respectively, in SiHa and HeLa cells after si-LINC01006 or nonsense sequence (si-NC) transfection. (G and H) SiHa and HeLa cells were co-transfected with si-LINC01006 and anti-miR-28-5p or anti-NC, and the mRNA and protein expression levels of PAK2 were measured. (I) RIP assays were performed to analyze the interaction between LINC01006, miR-28-5p, and PAK2; the relative enrichment of LINC01006, miR-28-5p and PAK2 in the immunoprecipitate was detected by RT-qPCR. (J) The mRNA expression level of PAK2 in 67 pairs of cervical cancer tissues and matched adjacent normal tissues was tested by RT-qPCR. (K) Pearson's correlation coefficient was used to analyze the expression correlation between miR-28-5p and PAK2 in cervical cancer tissues. (L) The correlation between the expression levels of LINC01006 and PAK2 in cervical cancer tissues was determined using Pearson's correlation coefficient. ${ }^{* *} \mathrm{P}<0.01$ vs. respective control. LINC01006, long intergenic non-protein-coding RNA 1006. RIP, radioimmunoprecipitation.

carcinogenesis and cancer progression (24-26). Considering the importance of IncRNAs, it is essential to examine the roles of cancer-related lncRNAs in the malignancy of cervical cancer and elucidate the underlying mechanisms, which are of the utmost importance for the development of attractive targets for cancer diagnosis, prognosis, and management. Over 50,000 lncRNAs are present in the human genome (27); however, the majority of these have not been studied in cervical cancer and thus require clarification. Therefore, the present study exam- ined the precise roles of LINC01006 in cervical cancer and determined its regulatory mechanism.

LINC01006 is upregulated in prostate (20) and pancreatic (21) cancers and plays pro-oncogenic roles. By contrast, this lncRNA is weakly expressed in gastric cancer and exhibits a notable association with age, tumor location, tumor size and venous invasion (22). These observations indicate tissue specificity in the expression profile and function of LINC01006 in human cancers. However, the expression pattern 

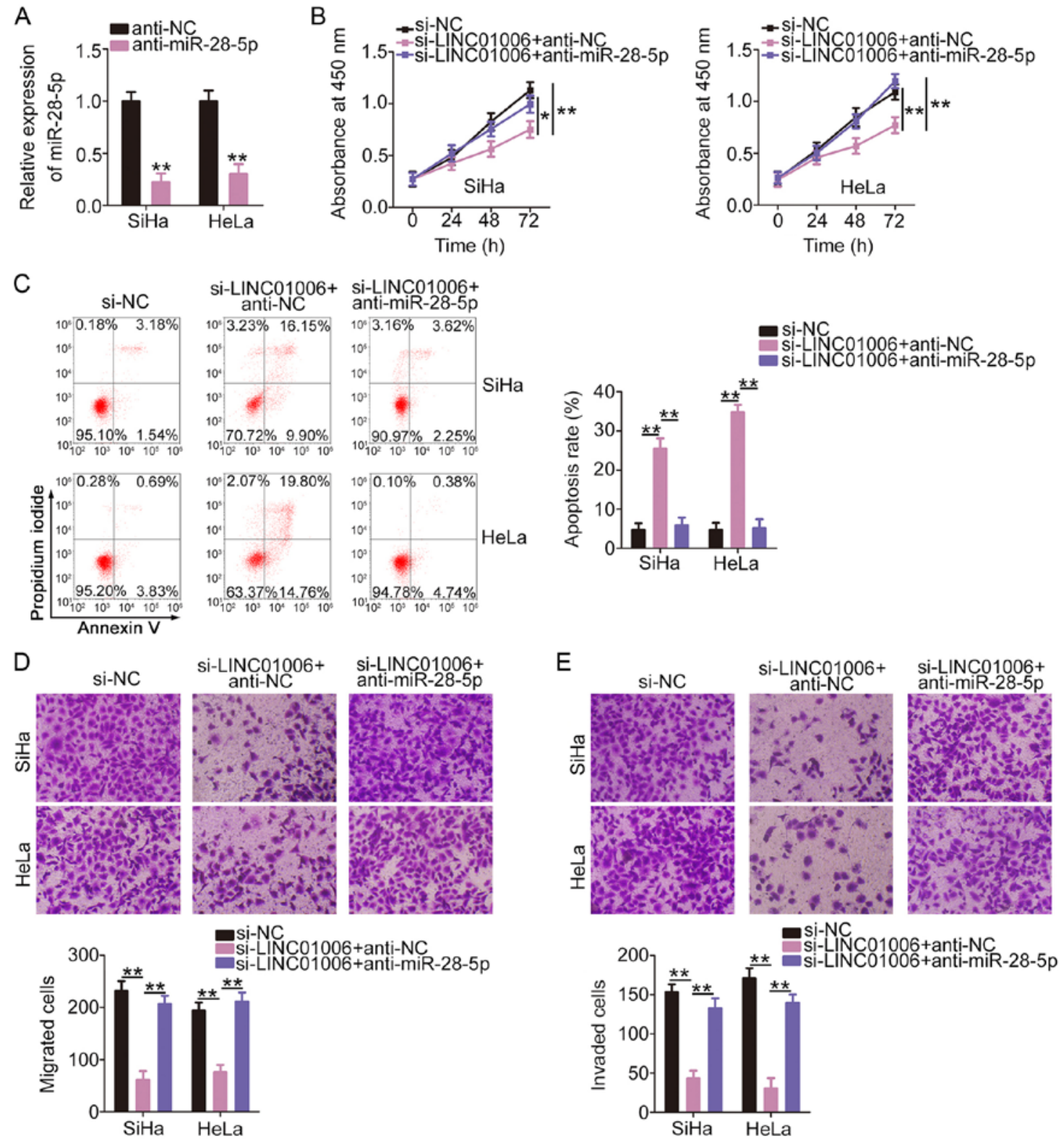

Figure 5. A miR-28-5p inhibitor (anti-miR-28-5p) offsets the inhibitory actions of LINC01006 silencing in cervical cancer cells. (A) miR-28-5p expression was detected in anti-miR-28-5p-transfected or NC inhibitor (anti-NC)-transfected SiHa and HeLa cells by RT-qPCR. (B and C) LINC01006-silenced SiHa and HeLa cells were transfected with anti-miR-28-5p or anti-NC. Cell proliferation and apoptosis were monitored using the cell proliferation assay and flow cytometric analysis, respectively. (D and E) The migratory and invasive abilities of the aforementioned cells were investigated using Transwell migration and invasion assays. ${ }^{*} \mathrm{P}<0.05$ and ${ }^{* *} \mathrm{P}<0.01$ vs. respective control. LINC01006, long intergenic non-protein-coding RNA 1006.

and detailed roles of LINC01006 in cervical cancer remain largely ambiguous. The present study demonstrated that the expression level of LINC01006 was visibly higher in cervical cancer tissues and cell lines. Survival analysis confirmed that a high LINC01006 expression was significantly associated with a worse overall survival of patients with cervical cancer. The silencing of LINC01006 suppressed the proliferation, migration and invasion of cervical cancer cells, but induced cell apoptosis in vitro. The absence of LINC01006 impeded tumor growth in vivo. Taken together, these results highlight LINC01006 as a potential target for cervical cancer diagnosis, prognosis and therapy.

The ceRNA theory was recently established, and it describes ceRNAs as a novel group of post-transcriptional regulators that participate in tumorigenesis and tumor devel- opment $(28,29)$. The ceRNA network involving lncRNAs, miRNAs and mRNAs is a widely accepted mechanism of post-transcriptional regulation of IncRNAs (30). Notably, the subcellular distribution of $1 n c R N A s$ determines their roles; i.e., whether an IncRNA functions as a ceRNA is largely based on its localization (31). IncRNAs that are primarily located in the nucleus generally affect gene expression at the pre-transcriptional or transcriptional levels (32). By contrast, cytoplasmic lncRNAs sequester certain miRNAs via the same miRNA response elements to indirectly modulate mRNA expression at the post-transcriptional level (33). The majority of LINC01006 expression was observed in the cytoplasm of cervical cancer cells in the present study. Therefore, it was hypothesized that LINC01006 exerts its tumor-promoting actions in cervical cancer in a ceRNA manner. 
A
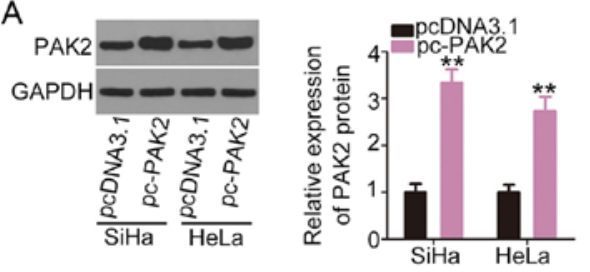

B
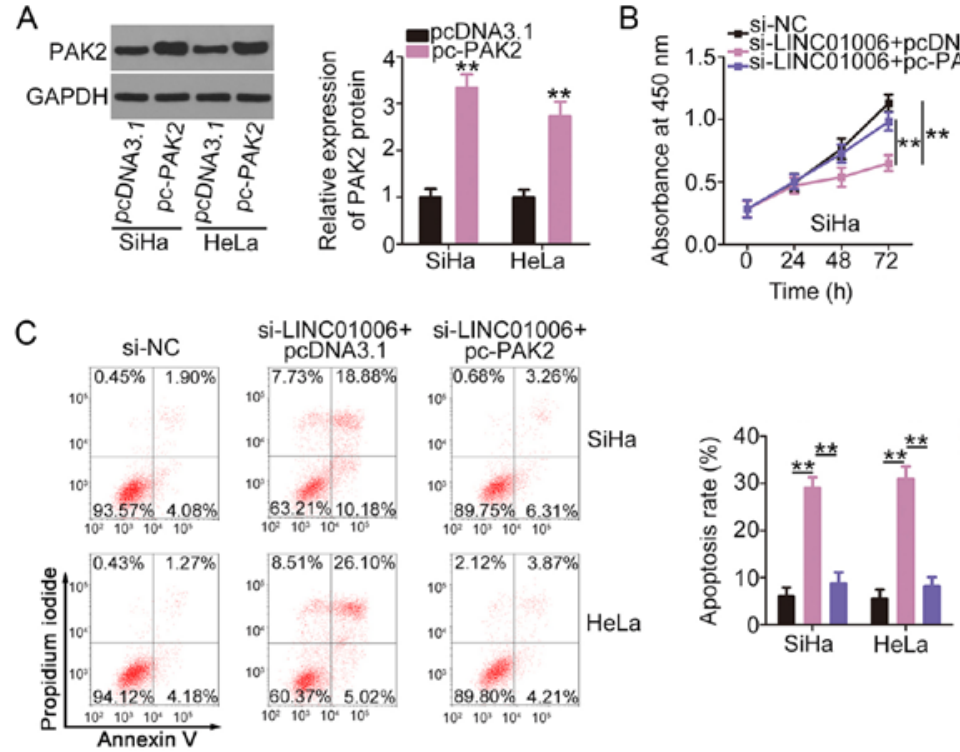

$8.51 \% 26.10 \%$
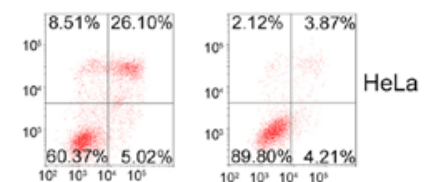

D si-NC

Si-LINCO1000+
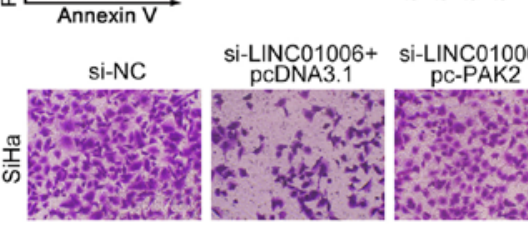
pCDNA3.
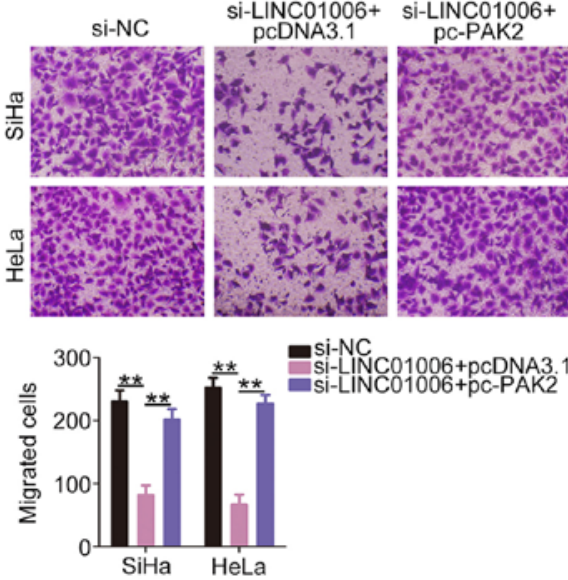

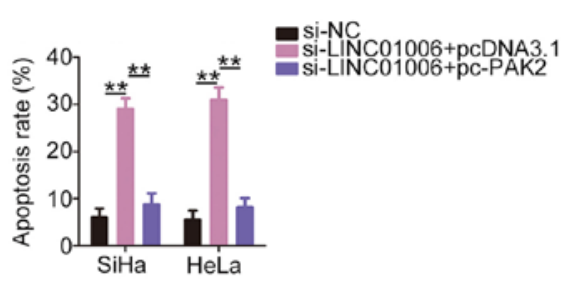

E
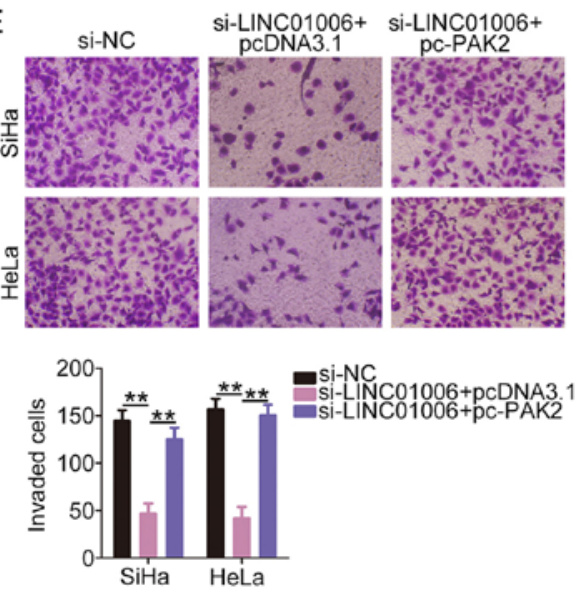

Figure 6. PAK2 upregulation partially reverses si-LINC01006-induced effects in cervical cancer cells. (A) Western blot analysis was used to measure the protein expression levels of PAK2 in SiHa and HeLa cells transfected with pc-PAK2 or pcDNA3.1. (B-E) si-LINC01006 in parallel with pc-PAK2 or pcDNA3.1 was transfected into $\mathrm{SiHa}$ and HeLa cells. Cell proliferation, apoptosis, migration and invasion were determined by cell proliferation assay, flow cytometric analysis, and Transwell migration and invasion assays, respectively. ${ }^{* *} \mathrm{P}<0.01$ vs. respective control. PAK2, P21-activated kinase 2 ; LINC01006, long intergenic non-protein-coding RNA 1006.

\section{A}
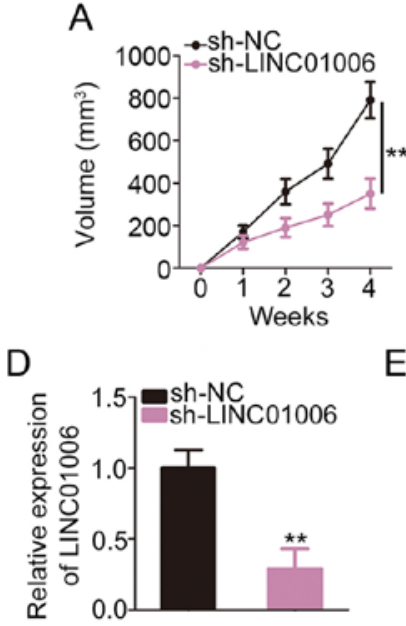

$\mathrm{E}$
B
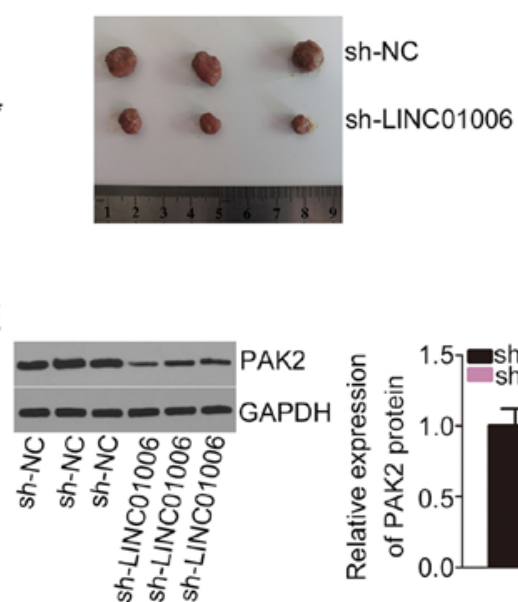

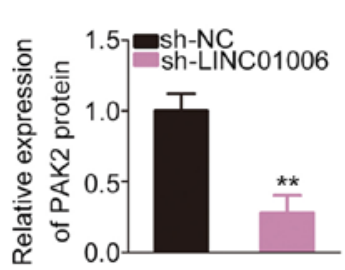

$\mathrm{F}$

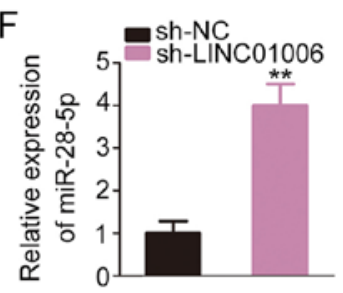

Figure 7. LINC01006 silencing hinders tumor growth in vivo. (A) Growth curves of the sh-LINC01006 and sh-NC groups were plotted based on the tumor volume detected weekly. (B) Representative morphologies of the tumor xenografts from the sh-LINC01006 and sh-NC groups were obtained at week 4. (C) At the end of the experiment, tumor xenografts were resected and weighed. (D and E) LINC01006 and PAK2 protein expression in mouse tumors obtained from the sh-LINC01006 and sh-NC groups was measured by RT-qPCR and western blot analysis, respectively. (F) miR-28-5p expression in tumor xenografts was analyzed using RT-qPCR. ${ }^{* *} \mathrm{P}<0.01$ vs. respective control. PAK2, P21-activated kinase 2; LINC01006, long intergenic non-protein-coding RNA 1006. 
Data from bioinformatics analysis suggested a possible binding interaction between LINC01006 and miR-28-5p. To test this hypothesis, luciferase reporter and RIP assays were performed. The results confirmed that LINC01006 physically interacted with miR-28-5p and sponged miR-28-5p in cervical cancer. Emerging studies have reported that miR-28-5p is differentially expressed in multiple human cancer types and plays an important role in tumorigenesis (34-36). Consistently, the data of the present study revealed the antioncogenic roles of miR-28-5p in cervical cancer. Additional in-depth mechanistic analyses revealed that PAK2 was a direct target of miR-28-5p. LINC01006 deficiency reduced the expression of PAK2 in cervical cancer cells, and this regulatory effect was achieved by sponging miR-28-5p. Notably, 3 molecules, namely, LINC01006, miR-28-5p and PAK2, co-existed in the same RNA-induced silencing complex. Correlation analysis revealed that PAK2 negatively correlated with miR-28-5p, but positively correlated with LINC01006 expression in cervical cancer tissues. The present study demonstrated a novel ceRNA pathway comprised of LINC01006, miR-28-5p and PAK2 in cervical cancer.

PAKs are a family of serine/threonine kinases that constitute 2 distinct subgroups: Subgroup 1 contains PAK 1-3 and subgroup 2 contains PAKs (37). PAK2 has an autoinhibitory domain and may be activated by the small GTP-binding proteins Cdc42 and Rac (38). Several studies have confirmed the implication of PAK2 in the course of cancer oncogenesis and progression (39-41). The downregulation of PAK2 by miRNAs abates the aggressiveness of human cancer cells. The present study observed a similar trend in cervical cancer. The final rescue experiments demonstrated that suppression of miR-28-5p or PAK2 overexpression abrogated the impacts of LINC01006 downregulation on malignant cellular functions in cervical cancer. Jointly, these results confirm that the miR-28-5p/PAK2 axis is a crucial mediator in which LINC01006 functions as a novel carcinogenic lncRNA in cervical cancer.

The present study revealed that LINC01006 increased PAK2 expression by acting as a ceRNA for miR-28-5p, which aggravated the oncogenicity of cervical cancer cells. These findings offer a basis for the identification of LINC01006-targeted clinical therapy.

\section{Acknowledgements}

Not applicable.

\section{Funding}

The present study was supported by the Renmin Hospital of Wuhan University.

\section{Availability of data and materials}

The datasets used and/or analyzed during the present study are available from the corresponding author on reasonable request.

\section{Authors' contributions}

All authors (LT, FH, JY, XM and LC) made significant contributions to the findings, analysis and methodology of the study. All authors read and approved the final draft.

\section{Ethics approval and consent to participate}

The Ethics Committee of Renmin Hospital of Wuhan University approved the present study. All experiments involving human samples were performed in accordance with the principles of the Declaration of Helsinki. All participants provided written informed consent. The Institutional Animal Care and Use Committee of Renmin Hospital of Wuhan University approved the experimental steps involving animals.

\section{Patient consent for publication}

Not applicable.

\section{Competing interests}

The authors declare that they have no competing interests.

\section{References}

1. Torre LA, Bray F, Siegel RL, Ferlay J, Lortet-Tieulent J and Jemal A: Global cancer statistics, 2012. CA Cancer J Clin 65: 87-108, 2015

2. Bray F, Ferlay J, Soerjomataram I, Siegel RL, Torre LA and Jemal A: Global cancer statistics 2018: GLOBOCAN estimates of incidence and mortality worldwide for 36 cancers in 185 countries. CA Cancer J Clin 68: 394-424, 2018.

3. Fang J, Zhang H and Jin S: Epigenetics and cervical cancer: From pathogenesis to therapy. Tumour Biol 35: 5083-5093, 2014.

4. Tsikouras P, Zervoudis S, Manav B, Tomara E, Iatrakis G, Romanidis C, Bothou A and Galazios G: Cervical cancer: Screening, diagnosis and staging. J BUON 21: 320-325, 2016.

5. Lin M, Ye M, Zhou J, Wang ZP and Zhu X: Recent advances on the molecular mechanism of cervical carcinogenesis based on systems biology technologies. Comput Struct Biotechnol J 17: 241-250, 2019.

6. Sarfi M, Abbastabar M and Khalili E: Long noncoding RNAs biomarker-based cancer assessment. J Cell Physiol 234: 16971-16986, 2019.

7. Ransohoff JD, Wei Y and Khavari PA: The functions and unique features of long intergenic non-coding RNA. Nat Rev Mol Cell Biol 19: 143-157, 2018

8. Shi X, Sun M, Wu Y, Yao Y, Liu H, Wu G, Yuan D and Song Y: Post-transcriptional regulation of long noncoding RNAs in cancer. Tumour Biol 36: 503-513, 2015.

9. Ponting CP, Oliver PL and Reik W: Evolution and functions of long noncoding RNAs. Cell 136: 629-641, 2009.

10. Ramakrishnaiah Y, Kuhlmann L and Tyagi S: Towards a comprehensive pipeline to identify and functionally annotate long noncoding RNA (lncRNA). Comput Biol Med 127: 104028, 2020.

11. Luo F, Wen Y, Zhou H and Li Z: Roles of long non-coding RNAs in cervical cancer. Life Sci 256: 117981, 2020.

12. He J, Huang B, Zhang K, Liu M and Xu T: Long non-coding RNA in cervical cancer: From biology to therapeutic opportunity. Biomed Pharmacother 127: 110209, 2020.

13. Galvão $M$ and Coimbra EC: Long noncoding RNAs (lncRNAs) in cervical carcinogenesis: New molecular targets, current prospects. Crit Rev Oncol Hematol 156: 103111, 2020.

14. Acunzo M, Romano G, Wernicke D and Croce CM: MicroRNA and cancer-a brief overview. Adv Biol Regul 57: 1-9, 2015.

15. Adams BD, Kasinski AL and Slack FJ: Aberrant regulation and function of microRNAs in cancer. Curr Biol 24: R762-R776, 2014.

16. Miao J, Regenstein JM, Xu D, Zhou D, Li H, Zhang H, Li C, Qiu $\mathrm{J}$ and Chen X: The roles of microRNA in human cervical cancer. Arch Biochem Biophys 690: 108480, 2020.

17. Shen S, Zhang S, Liu P, Wang J and Du H: Potential role of microRNAs in the treatment and diagnosis of cervical cancer. Cancer Genet 248-249: 25-30, 2020.

18. Tornesello ML, Faraonio R, Buonaguro L, Annunziata C, Starita N, Cerasuolo A, Pezzuto F, Tornesello AL and Buonaguro FM: The role of microRNAs, long non-coding RNAs, and circular RNAs in cervical cancer. Front Oncol 10: 150, 2020. 
19. Ye Y, Shen A and Liu A: Long non-coding RNA H19 and cancer: A competing endogenous RNA. Bull Cancer 106: 1152-1159, 2019.

20. Ma E, Wang Q, Li J, Zhang X, Guo Z and Yang X: LINC01006 facilitates cell proliferation, migration and invasion in prostate cancer through targeting miR-34a-5p to up-regulate DAAM1. Cancer Cell Int 20: 515, 2020.

21. Zhang L, Wang Y, Zhang L, You G, Li C, Meng B, Zhou M and Zhang M: LINC01006 promotes cell proliferation and metastasis in pancreatic cancer via miR-2682-5p/HOXB8 axis. Cancer Cell Int 19: 320, 2019.

22. Zhu X, Chen F, Shao Y, Xu D and Guo J: Long intergenic non-protein coding RNA 1006 used as a potential novel biomarker of gastric cancer. Cancer Biomark 21: 73-80, 2017.

23. Livak KJ and Schmittgen TD: Analysis of relative gene expression data using real-time quantitative PCR and the 2(-Delta Delta C(T)) method. Methods 25: 402-408, 2001.

24. Duan DM, Zhang L and Hua F: LncRNA UCA1 inhibits proliferation and promotes apoptosis of cervical cancer cells by regulating beta-catenin/TCF-4. Eur Rev Med Pharmacol Sci 24 5963-5969, 2020.

25. Yan A, Chen G and Nie J: DGUOK-AS1 promotes the proliferation cervical cancer through regulating miR-653-5p/EMSY. Cancer Biol Ther 1-9, 2020.

26. Lin L, Xin B, Jiang T, Wang XL, Yang H and Shi TM: Long non-coding RNA LINC00460 promotes proliferation and inhibits apoptosis of cervical cancer cells by targeting microRNA-503-5p Mol Cell Biochem 475: 1-13, 2020.

27. Xu J, Bai J, Zhang X, Lv Y, Gong Y, Liu L, Zhao H, Yu F, Ping Y, Zhang G, et al: A comprehensive overview of lncRNA annotation resources. Brief Bioinform 18: 236-249, 2017.

28. Niu ZS, Wang WH, Dong XN and Tian LM: Role of long noncoding RNA-mediated competing endogenous RNA regulatory network in hepatocellular carcinoma. World J Gastroenterol 26: 4240-4260, 2020.

29. Raziq K, Cai M, Dong K, Wang P, Afrifa J and Fu S: Competitive endogenous network of IncRNA, miRNA, and mRNA in the chemoresistance of gastrointestinal tract adenocarcinomas. Biomed Pharmacother 130: 110570, 2020.

30. Zhang XZ, Liu H and Chen SR: Mechanisms of long non-coding RNAs in cancers and their dynamic regulations. Cancers (Basel) 12: 1245, 2020.

31. Du H and Chen Y: Competing endogenous RNA networks in cervical cancer: Function, mechanism and perspective. J Drug Target 27: 709-723, 2019.
32. Zhang K, Shi ZM, Chang YN, Hu ZM, Qi HX and Hong W: The ways of action of long non-coding RNAs in cytoplasm and nucleus. Gene 547: 1-9, 2014.

33. Rashid F, Shah A and Shan G: Long non-coding RNAs in the cytoplasm. Genomics Proteomics Bioinformatics 14: 73-80, 2016.

34. Ma L, Zhang Y and Hu F: miR285p inhibits the migration of breast cancer by regulating WSB2. Int J Mol Med 46: 1562-1570, 2020.

35. Zhang L, Wang X, Liu X, Lv M, Shen E, Zhu G and Sun Z: miR-28-5p targets MTSS1 to regulate cell proliferation and apoptosis in esophageal cancer. Acta Biochim Biophys Sin (Shanghai) 52: 842-852, 2020.

36. Fazio S, Berti G, Russo F, Evangelista M, D'Aurizio R, Mercatanti A, Pellegrini M and Rizzo M: The miR-28-5p targetome discovery identified SREBF2 as one of the mediators of the miR-28-5p tumor suppressor activity in prostate cancer cells. Cells 9: 354, 2020

37. Nuche-Berenguer B, Ramos-Alvarez I and Jensen RT: The p21-activated kinase, PAK2, is important in the activation of numerous pancreatic acinar cell signaling cascades and in the onset of early pancreatitis events. Biochim Biophys Acta 1862: 1122-1136, 2016

38. Knaus UG, Wang Y, Reilly AM, Warnock D and Jackson JH: Structural requirements for PAK activation by Rac GTPases. J Biol Chem 273: 21512-21518, 1998

39. Liu H, Shin SH, Chen H, Liu T, Li Z, Hu Y, Liu F, Zhang C, Kim DJ, Liu K and Dong Z: CDK12 and PAK2 as novel therapeutic targets for human gastric cancer. Theranostics 10: 6201-6215, 2020 .

40. Lee JS, Mo Y, Gan H, Burgess RJ, Baker DJ, van Deursen JM and Zhang Z: Pak2 kinase promotes cellular senescence and organismal aging. Proc Natl Acad Sci USA 116: 13311-13319, 2019.

41. Gupta A, Ajith A, Singh S, Panday RK, Samaiya A and Shukla S: PAK2-c-Myc-PKM2 axis plays an essential role in head and neck oncogenesis via regulating Warburg effect. Cell Death Dis 9: 825,2018

This work is licensed under a Creative Commons Attribution-NonCommercial-NoDerivatives 4.0 International (CC BY-NC-ND 4.0) License. 